\title{
Increased interference fringe visibility from the post-fabrication heat treatment of a perfect crystal silicon neutron interferometer
}

B. Heacock, M. Arif, D. G. Cory, T. Gnaeupel-Herold, R. Haun, M. G. Huber, M. E. Jamer, J. Nsofini, D. A. Pushin, D. Sarenac, I. Taminiau, and A. R. Young

Citation: Review of Scientific Instruments 89, 023502 (2018); doi: 10.1063/1.5008273

View online: https://doi.org/10.1063/1.5008273

View Table of Contents: http://aip.scitation.org/toc/rsi/89/2

Published by the American Institute of Physics

\section{Articles you may be interested in}

Noise refocusing in a five-blade neutron interferometer

Journal of Applied Physics 122, 054501 (2017); 10.1063/1.4996866

Simple, low-noise piezo driver with feed-forward for broad tuning of external cavity diode lasers

Review of Scientific Instruments 89, 023102 (2018); 10.1063/1.5009643

Scanning microwave microscopy applied to semiconducting GaAs structures

Review of Scientific Instruments 89, 023704 (2018); 10.1063/1.5015966

Design of a multiband near-infrared sky brightness monitor using an InSb detector

Review of Scientific Instruments 89, 023107 (2018); 10.1063/1.5010819

Quantitative characterization of semiconductor structures with a scanning microwave microscope

Review of Scientific Instruments 89, 023706 (2018); 10.1063/1.5013113

A variable partially polarizing beam splitter

Review of Scientific Instruments 89, 023108 (2018); 10.1063/1.5004805

This article may be downloaded for personal use only. Any other use requires prior permission of the author and AIP Publishing. The following article appeared in Heacock, B., Arif, M., Cory, D. G., Gnaeupel-Herold, T., Haun, R., Huber, M. G., ... Young, A. R. (2018). Increased interference fringe visibility from the post-fabrication heat treatment of a perfect crystal silicon neutron interferometer. Review of Scientific Instruments, 89(2), 023502.

doi:10.1063/1.5008273 and may be found at https://doi.org/10.1063/1.5008273

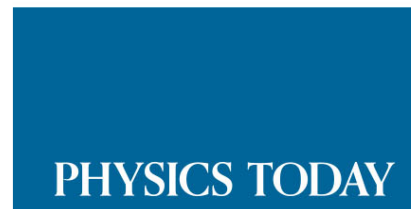

MANAGER'S GUIDE

WHITEPAPERS
Accelerate R\&D with

Multiphysics Simulation
READ NOW

PRESENTED BY

V СОMSOL 


\title{
Increased interference fringe visibility from the post-fabrication heat treatment of a perfect crystal silicon neutron interferometer
}

\author{
B. Heacock, ${ }^{1,2, a)}$ M. Arif, ${ }^{3}$ D. G. Cory, ${ }^{4,5,6,7}$ T. Gnaeupel-Herold, ${ }^{3}$ R. Haun, ${ }^{8}$ M. G. Huber, ${ }^{3}$ \\ M. E. Jamer, ${ }^{3}$ J. Nsofini, ${ }^{4,9}$ D. A. Pushin, ${ }^{4,9}$ D. Sarenac,,${ }^{4,9}$ I. Taminiau, ${ }^{10}$ and A. R. Young ${ }^{1,2}$ \\ ${ }^{1}$ Department of Physics, North Carolina State University, Raleigh, North Carolina 27695, USA \\ ${ }^{2}$ Triangle Universities Nuclear Laboratory, Durham, North Carolina 27708, USA \\ ${ }^{3}$ National Institute of Standards and Technology, Gaithersburg, Maryland 20899, USA \\ ${ }^{4}$ Institute for Quantum Computing, University of Waterloo, Waterloo, Ontario N2L3G1, Canada \\ ${ }^{5}$ Department of Chemistry, University of Waterloo, Waterloo, Ontario N2L3G1, Canada \\ ${ }^{6}$ Perimeter Institute for Theoretical Physics, Waterloo, Ontario N2L2Y5, Canada \\ ${ }^{7}$ Canadian Institute for Advanced Research, Toronto, Ontario M5G 1Z8, Canada \\ ${ }^{8}$ Department of Physics, Tulane University, New Orleans, Louisiana 70118, USA \\ ${ }^{9}$ Department of Physics, University of Waterloo, Waterloo, Ontario N2L3G1, Canada \\ ${ }^{10}$ Quantum Valley Investments, Waterloo, Ontario N2L OA9, Canada
}

(Received 4 October 2017; accepted 18 January 2018; published online 8 February 2018)

\begin{abstract}
We find that annealing a previously chemically etched interferometer at $800^{\circ} \mathrm{C}$ dramatically increased the interference fringe visibility from $23 \%$ to $90 \%$. The Bragg plane misalignments were also measured before and after annealing using neutron rocking curves, showing that Bragg plane alignment was improved across the interferometer after annealing. This suggests that current interferometers with low fringe visibility may be salvageable and that annealing may become an important step in the fabrication process of future neutron interferometers, leading to less need for chemical etching and larger more exotic neutron interferometers. Published by AIP Publishing. https://doi.org/10.1063/1.5008273
\end{abstract}

\section{INTRODUCTION}

Perfect silicon neutron interferometers (see Fig. 1) coherently split and recombine an incoming neutron beam using a series of Bragg diffractions. The macroscopic separation of the beam paths has led to many historically important experiments over the last 40 years, including demonstrations of gravitational quantum interference, the $4 \pi$ periodicity of Dirac spinors, violation of Bell-like inequalities, phase and contrast imaging, neutron holography, and more. ${ }^{1-10}$ For a history of the field, see Ref. 11. The required relative Bragg plane alignment of the splitter, mirror, and analyzer diffracting crystals (labeled as S, M, and A, respectively in Fig. 2) has only ever been achieved by cutting neutron interferometers from a single float zone grown silicon ingot using a rotating diamond saw, leaving splitter, mirror, and analyzer crystal "blades" protruding from a common base. The interferometer is then etched in a mixture of hydrofluoric, nitric, and sometimes acetic acids. For a good description of interferometer fabrication, see Ref. 12.

Neutron interferometers are typically etched iteratively by removing 10 s of microns with each etch and then checking contrast by rotating a flat piece of fused silica between the splitter and mirror or mirror and analyzer blades. The resulting sinusoidal neutron interference signal can theoretically have $100 \%$ contrast in the O-Beam [Fig. 2(a)], where the contrast is given by the amplitude over the mean of the fitted oscillation. Etching is believed to relieve the strain in the crystal caused by the machining damage. ${ }^{12}$ However, as the total etching depth increases, the parallelism and uniform thicknesses of the

a)Electronic mail: bjheacoc@ncsu.edu crystal blades degrade due to the uneven etch rates. If too much material is etched away, the contrast begins to drop. Additionally, it is well established that the neutron interferometer contrast varies depending on where the incident beam strikes the splitter blade and for different wavelengths. We recently measured a variation in Bragg plane alignment across a $1 \mathrm{~cm}$ span of the splitter blade of another interferometer of up to $40 \mathrm{nrad},{ }^{13}$ a phenomenon suggested earlier in Ref. 14. This finding as well as a variation in the blade thicknesses across the particular interferometer used in this experiment is confirmed here.

Interferometers constructed under similar conditions can show wildly different contrasts. Previously, machining accuracy was thought to be a major source of this variation. However, modern machining processes eliminate this as a possibility. This work demonstrates that the lack of reproducibility in interferometer construction is likely due to the thickness variations of the interferometer blades from uneven etch rates as well as fluctuating or large (greater than $10 \mathrm{nrad}$ ) Bragg plane misalignments between the blades. We show that Bragg plane misalignment can be reduced by annealing the interferometer after fabrication.

\section{BRAGG DIFFRACTION AND THE NEUTRON COHERENCE LENGTH}

Because neutron beams have a spread in momentum space that is much broader than the angular acceptance for Bragg scattering from a perfect silicon crystal, called the Darwin width $\Theta_{\mathrm{D}}$, the transverse coherence length of a Braggdiffracted neutron wavepacket is given by the pendellösung length, which is about $50 \mu \mathrm{m}$ for typical neutron wavelengths 


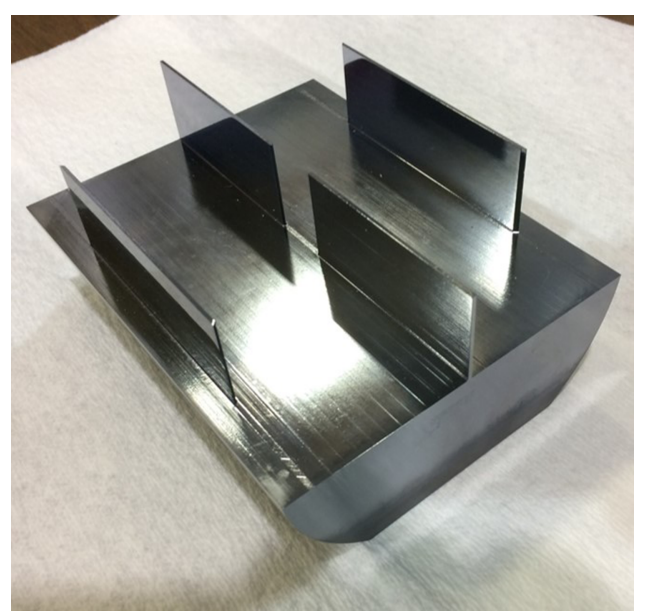

FIG. 1. The neutron interferometer annealed in this experiment. The base is roughly $10 \mathrm{~cm} \times 10 \mathrm{~cm}$. The blades are $3 \mathrm{~cm}$ tall.

and first order Bragg diffraction. Here the transverse direction is perpendicular to the Bragg planes and along the crystal blades. ${ }^{15}$ If machining inaccuracies or uneven etch rates cause the two beam paths in a neutron interferometer [Paths $|\mathrm{I}\rangle$ and $\mid$ III $\rangle$ in Fig. 2(a)] to be displaced relative to each other outside of the pendellösung length, then the interferometer contrast suffers. This sets the machining tolerances for the interferometer blades, indicating that the parallelism, thickness, and spacing of the crystal blades should be uniform to a level that is much smaller than the pendellösung length. ${ }^{11}$

Bragg plane misalignments between the diffracting blades of the interferometer can also cause its contrast to suffer due to the details of neutron dynamical Bragg diffraction by a perfect crystal. (For a complete description of dynamical diffraction, see Refs. 11, 16, and 17. Dynamical diffraction can also be approached from a quantum information perspective. ${ }^{18,19}$ ) For subsequent diffracting crystals of the same thickness, the Borrmann fan (the spreading of the diffracted neutron beam upon entering and exiting each blade is visible in Fig. 2) is coherently recombined, interfering constructively in the twice or more reflected beam. This creates a characteristic peak in the neutron intensity, which can be measured by rotating a refracting prism about the beam axis between the blades of an interferometer. The prism slightly deflects the beam, and rotating the prism moves the refraction plane of the prism in and out of the diffraction plane of the crystal. ${ }^{14,20,21}$ These peaks have an angular scale in the Bragg plane misalignment of $\delta=(H D)^{-1}$ $\sim 100$ nrad, where $H$ is the reciprocal lattice vector and $D$ is the crystal thickness. The same effect occurs in Mach-Zehnder neutron interferometers, where it dephases the interferometer and sets the misalignment tolerances of the Bragg planes in each blade. For a generalized description of how the interferometer contrast is affected by Bragg plane misalignments, see Ref. 13. Additionally, a computational program outlined in Refs. 22 and 23 is able to accommodate the thickness differences and different incoming momentum space beam profiles in a neutron interferometer.

To characterize the impact of annealing on Bragg plane alignment of the interferometer blades, we measured the oscillatory rocking curve structure of multiple Bragg diffractions
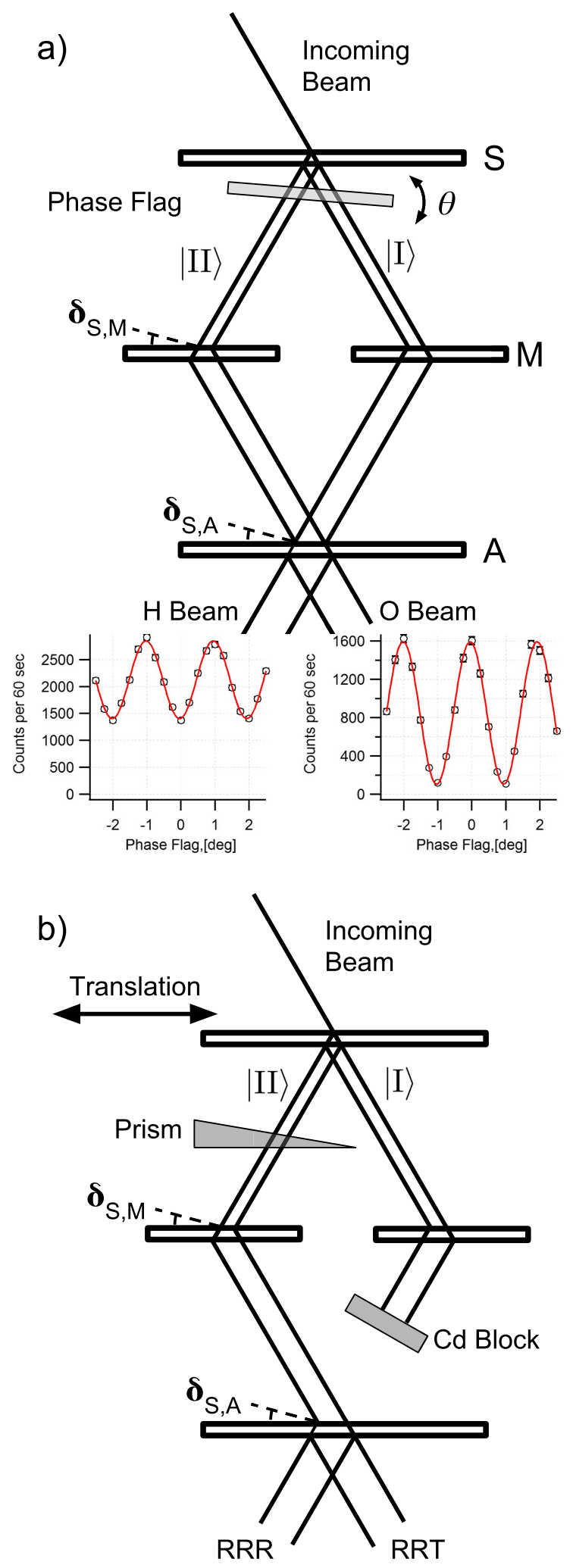

FIG. 2. The geometries used in this experiment. (a) Rotating a phase flag, $\theta$, in the interferometer generates a sinusoidal signal. Shown in (b) is the geometry used to measure the misalignment and thickness variation in the interferometer. The rocking curve oscillatory structures upon rotating the prism about the beam axis are shown in Figs. 4 and 5. Adapted from Heacock et al., Phys. Rev. A 95, 013840 (2017). Copyright 2017 American Physical Society.

in one arm of the interferometer as a function of rotation of a fused silica prism. ${ }^{13,14,20,21}$ In this way, we can directly see the relative Bragg plane alignment change of the interferometer 
blades by about 100 nrad after annealing as a shift in the diffracted beam intensity peak position. The structure of each peak is perturbed by unequal crystal thicknesses on the pendellösung length scale, and we find that the overall structure of each peak does not change with annealing. We are thus able to differentiate between the detrimental effects of unequal blade thicknesses and the Bragg plane misalignments in the interferometer and show that the post-fabrication annealing of the interferometer can improve the latter.

\section{EXPERIMENT}

This work was performed at the National Institute of Standards and Technology (NIST) Center for Neutron Research (NCNR). There are two dedicated interferometry beamlines at the NCNR. One beamline uses a $2.7 \AA$ neutron wavelength and a sophisticated vibration isolation system, the details of which can be found in Refs. 11 and 24. The second beamline has $2.2 \AA$ and $4.4 \AA$ neutron wavelengths available. A description of the second beamline can be found in Refs. 25 and 26. Both beamlines and all three wavelengths were used in this experiment.

To begin, smaller float-zone $(\mathrm{Fz})$ grown silicon-crystal samples (see Fig. 3) were annealed at a variety of temperatures before attempting to anneal the much larger interferometer crystal. The samples were cut using a rotating diamond saw with a fine grit size, but none of the samples were etched. These samples were tested with both $\mathrm{x}$-rays and neutrons. Unlike the silicon crystals grown by the Czochralski $(\mathrm{Cz})$ method, whose oxygen content causes strain in the crystal structure to increase with annealing, crystal planes become more highly ordered with annealing for Fz crystals. ${ }^{27} \mathrm{X}$-ray stress analysis of the crystal surfaces was performed on several of the samples using the technique described in Ref. 28. These results are summarized in Table I, and one can see the reduction of surface stresses in the crystals after annealing.

The samples were annealed in a tabletop tube furnace. The annealing process consisted of placing the sample inside an evacuated $25.2 \mathrm{~mm}$ diameter quartz tube furnace. The temperature was ramped at a rate of $1{ }^{\circ} \mathrm{C} / \mathrm{min}$ up to a set constant temperature. The sample was held at the set temperature for several hours, and then the temperature was ramped down at the same $1{ }^{\circ} \mathrm{C} / \mathrm{min}$ rate. The interferometer was annealed in a larger tube furnace with an inner diameter of $208 \mathrm{~mm}$ under a constant argon flow at $800{ }^{\circ} \mathrm{C}$ for $10 \mathrm{~h}$, not including the

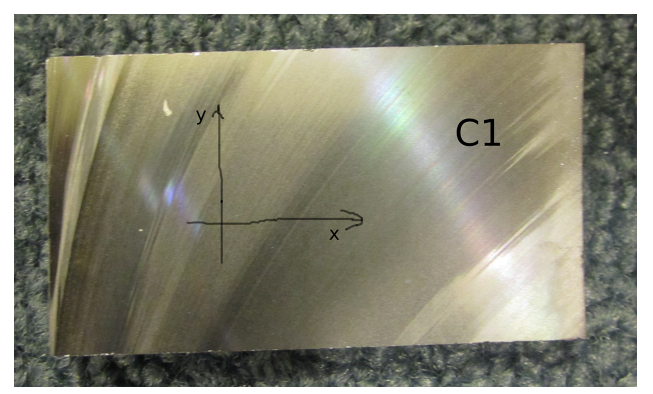

FIG. 3. The sample crystal \#1. The coordinate scale denotes the surface stress analysis orientation. The crystal dimensions are $43 \mathrm{~mm} \times 25 \mathrm{~mm} \times 4 \mathrm{~mm}$.
TABLE I. The results of the x-ray surface stress analysis $\sigma_{x x}$ and $\sigma_{y y}$ on several test samples. If an annealing temperature is not provided, the measurement was made before the crystal was annealed. The uncertainties are indicated in the brackets. The full width at half maximum (FWHM) data are from fitting the $\mathrm{Cu} \mathrm{K} \alpha_{1}$ x-ray diffraction peak to a Voigt function. The FWHM measurements were performed using a separate diffractometer.

\begin{tabular}{lccccc}
\hline \hline Sample & Description & $\begin{array}{c}\sigma_{x x} \\
(\mathrm{MPa})\end{array}$ & $\begin{array}{c}\sigma_{y y} \\
(\mathrm{MPa})\end{array}$ & $\begin{array}{c}\text { Anneal } \\
T\left({ }^{\circ} \mathrm{C}\right)\end{array}$ & $\begin{array}{c}\text { FWHM } \\
(\operatorname{arcsec})\end{array}$ \\
\hline Crystal \#1 & Diamond saw & $2(3)$ & $-121(3)$ & $\ldots$ & $\ldots$ \\
Crystal \#1 & Diamond saw & $-10(3)$ & $12(5)$ & 900 & $\ldots$ \\
Crystal \#2 & Diamond saw & $-1(4)$ & $17(9)$ & 700 & $\ldots$ \\
Crystal \#2 & Diamond saw & $6(3)$ & $6(5)$ & 900 & $\ldots$ \\
Crystal \#3 & Lapped & $\ldots$ & $\ldots$ & $\ldots$ & $168(3)$ \\
Crystal \#3 & Lapped & $\ldots$ & $\ldots$ & 800 & $102(3)$ \\
\hline \hline
\end{tabular}

ramping time. The ramping rate was $5{ }^{\circ} \mathrm{C} / \mathrm{min}$. Lapped Fz silicon crystal slabs $(142 \mathrm{~mm} \times 45 \mathrm{~mm} \times 6 \mathrm{~mm})$ were annealed in the furnace under the same conditions before attempting to anneal the interferometer. The full width at half maximum (FWHM) of the $\mathrm{Cu} \mathrm{K} \alpha_{1}$ diffraction peak of a lapped sample before and after annealing is shown in Table I with the $\mathrm{x}$-ray results from the smaller furnace.

The interferometer that was annealed uses the (111) Bragg reflection. In the past, the interferometer had exhibited a maximum contrast of $23 \%$ at $2.7 \AA .{ }^{29}$ Immediately before annealing, it was tested for contrast at $2.2 \AA$ and $4.4 \AA$, though no visible contrast was found. When searching for contrast, an interferometer is translated vertically and horizontally along the splitter blade, thus making a "contrast map" (see Fig. 7).

Before annealing, the interference structure of misaligning the mirror and analyzer blades, relative to the splitter, was studied by placing a fused silica prism between the splitter and mirror blades of the interferometer. The prism was placed so that its $6^{\circ}$ pitch was oriented at a right angle to the diffraction plane to within a few degrees. By then rotating the prism about the beam axis, deflection of the beam from the prism enters the diffraction plane, which causes the same effect as rotating the analyzer and mirror blades relative to the splitter blade at the nanoradian level. The structure associated with this rocking curve has been studied in the past. ${ }^{14,20,21}$

The angular deflection in the diffraction plane caused by the prism is

$$
\delta=\frac{\lambda^{2}}{2 \pi} \tan \alpha \sin \phi \sum_{i} N_{i} b_{i},
$$

where $\lambda$ is the neutron wavelength; $\alpha$ is the pitch of the prism; $\phi$ is the tilt of the prism about the beam axis; and the sum is over the number densities $N_{i}$ and scattering lengths $b_{i}$ of each species.

As the prism is rotated between the splitter and one of the mirror crystals with the other beam blocked, the reflectedreflected-transmitted (RRT) and reflected-reflected-reflected (RRR) beams are counted in ${ }^{3} \mathrm{He}$ detectors [Fig. 2(b)]. The position of the RRR peak is an average of the misalignment between the splitter and mirror blades and the splitter and analyzer blades. By adding the RRT and RRR beams together, we form the reflected-reflected (RR) beam, whose position is given by the Bragg plane misalignment between the splitter 
and mirror blades. The RR and RRR peaks can then be studied as a function of incident beam position by translating the interferometer along its blades using a positioning stage under the interferometer [Fig. 2(b)] and repeating the scans of prism rotation.

To describe the RR and RRR peaks, first we define two special functions:

$$
\begin{aligned}
& \mathcal{I}(\alpha, \beta)=\int_{-1}^{1} d \Gamma \sqrt{1-\Gamma^{2}} \cos (\alpha \Gamma) \cos \left(\frac{\beta}{\sqrt{1-\Gamma^{2}}}\right) \\
& \mathcal{J}(\alpha, \beta)=\int_{-1}^{1} d \Gamma\left(1-\Gamma^{2}\right)^{\frac{3}{2}} \cos (\alpha \Gamma) \cos \left(\frac{\beta}{\sqrt{1-\Gamma^{2}}}\right) .
\end{aligned}
$$

Here $\alpha$ is related to the peak position and Bragg plane misalignments; $\beta \neq 0$ is a correction to the standard results ${ }^{21}$ for unequal crystal thicknesses; and the integration over $\Gamma$ is an integration over the Borrmann fan. ${ }^{11}$ Note that for $\beta=0$ these functions can be written in terms of Bessel functions. ${ }^{21}$

The RR peak is given by

$$
I_{\mathrm{RR}}=A\left\{\pi+\mathcal{I}\left[B\left(\delta(\phi)+\delta_{S, M}^{\mathrm{rel}}\right), \Delta_{M, S}\right]\right\},
$$

where $A, B, \Delta_{M, S}$, and $\delta_{S, M}^{\text {rel }}$ are fit parameters; $\delta(\phi)$ is given by Eq. (1) as a function of prism rotation $\phi$. The RRR peak was fit to

$$
\begin{aligned}
I_{\mathrm{RRR}}= & A^{\prime}\left\{\frac{9}{16} \pi+\mathcal{J}\left[B\left(\delta(\phi)+\delta_{S, M}^{\mathrm{rel}}\right), \Delta_{M, S}^{\mathrm{rel}}\right]\right. \\
& +\mathcal{J}\left[B\left(\delta_{S, M}^{\mathrm{rel}}-\delta_{S, A}^{\mathrm{rel}}\right), \Delta_{A, S}-\Delta_{M, S}\right] \\
& \left.+\mathcal{J}\left[B\left(\delta(\phi)+\delta_{S, A}^{\mathrm{rel}}\right), \Delta_{A, S}\right]\right\},
\end{aligned}
$$

where $A^{\prime}, \Delta_{A, S}$, and $\delta_{S, A}^{\text {rel }}$ are fit parameters, and $B, \Delta_{M, S}$, and $\delta_{S, M}^{\mathrm{rel}}$ are the same parameters as those appearing in Eq. (4). For each incident beam position, global fits were performed by fitting $I_{\mathrm{RRR}}$ to the RRR beam intensity and $I_{\mathrm{RR}}-I_{\mathrm{RRR}}$ to the RRT beam intensity. All fits had $42-7$ degrees of freedom; the reduced $\chi^{2}$ values were between 0.6 and 1.1.

The $\Delta_{i, j}$ fit parameters are interpreted as the thickness difference between the splitter, mirror, and analyzer blades (subscripts $S, M$, and $A$, respectively) scaled by the pendellösung length:

$$
\Delta_{i, j}=2 \pi \frac{D_{i}-D_{j}}{\Delta_{H}},
$$

where $\Delta_{H}$ is the pendellösung length.

The $\delta_{i, j}^{\text {rel }}$ values are interpreted as the angular misalignments between the $i$ and $j$ interferometer blades (again labeled as $S, M$, or $A$ ), with an offset due to the unknown absolute alignment of the prism rotation $\phi$ with the diffraction plane of the interferometer. An offset in the readout of the prism's rotational positioning stage creates $\phi \rightarrow \phi+\phi_{0}$ in Eq. (1). The prism was rotationally aligned to the interferometer by eye, so the offset $\phi_{0}$ is less than $\sim \pm 10^{\circ}$ and Eq. (1) can be expanded such that $\delta(\phi) \rightarrow \delta(\phi)+\delta_{p}$, where $\delta_{p}=\delta\left(\phi_{0}\right)$. This creates the same constant offset $\delta_{p}$ for the $\delta_{S, M}^{\text {rel }}$ and $\delta_{S, A}^{\text {rel }}$ fit parameters. This offset is the same for the RR and RRR peaks both before and after annealing. The absolute Bragg plane alignment between the $i$ and $j$ blades is related to the fit parameters by

$$
\delta_{i, j}^{\mathrm{abs}}=\delta_{i, j}^{\mathrm{rel}}-\delta_{p}
$$

Note that the unknown values of $\delta_{i, j}^{\text {abs }}$ prevent the prism rotation $\phi$ from being aligned with neutrons. Finally, the absolute alignment between the mirror and analyzer blades is available by noting that

$$
\delta_{A, M}^{\mathrm{abs}}=\delta_{S, M}^{\mathrm{rel}}-\delta_{S, A}^{\mathrm{rel}} .
$$

Figures 4 and 5 show the fitted functions for a few incident beam positions on the interferometer at $4.4 \AA$. In addition to measuring Bragg plane misalignments, the structure of the peak lends information on the difference in thickness between the interferometer blades through the $\Delta_{i, j}$ fit parameters. This effect can be clearly seen in the widening and double peak structure in Fig. 4 as the interferometer is translated. The clearly visible gradient in the peak centroids in Fig. 5(a) before annealing is diminished after annealing. All curves shift to around 8 degrees of prism rotation after annealing, suggesting $\phi_{0} \sim 8^{\circ}$. The larger scatter in Figs. 4(b) and 5(b) is likely due at least in part to the shorter count times resulting in a higher error per point in the RR and RRR rocking curves.

These effects are all reflected in Fig. 6, where the peak centroids converted to units of Bragg plane misalignment are plotted. Absolute misalignments between the mirror and analyzer blades are shown in Fig. 6(b). Gradients in the Bragg plane misalignments drop from about $10 \mathrm{nrad} / \mathrm{mm}$ to less than $5 \mathrm{nrad} / \mathrm{mm}$ after annealing, and the absolute misalignment between the mirror and analyzer blades also lessens for

(a) Before Annealing

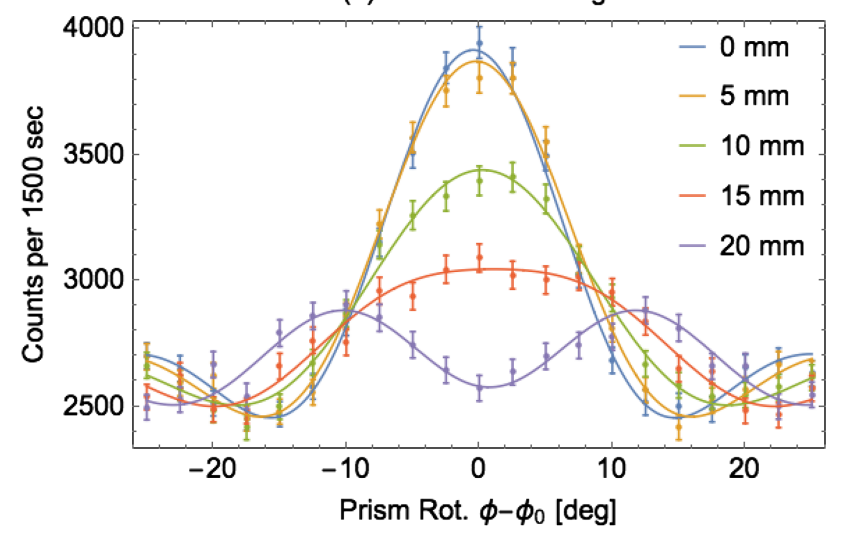

(b) After Annealing

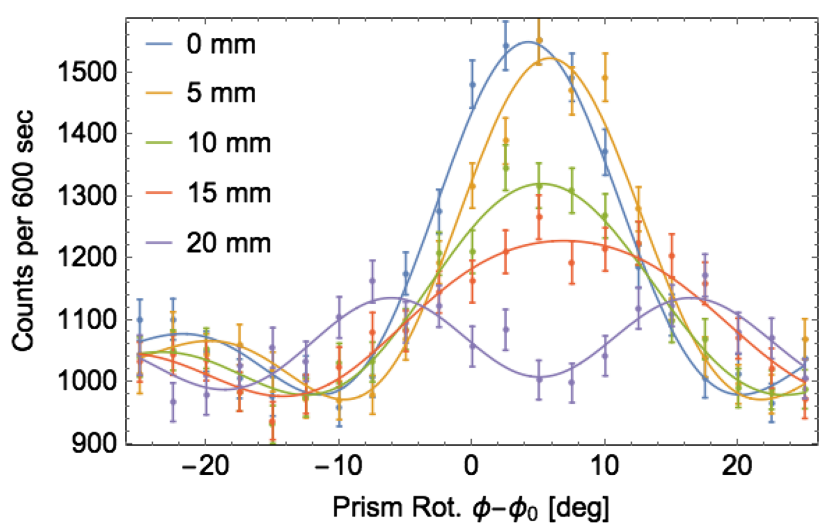

FIG. 4. The RR peaks before (a) and after (b) annealing with best fits. Each curve is for a different translation of the interferometer relative to the incoming beam. 
(a) Before Annealing

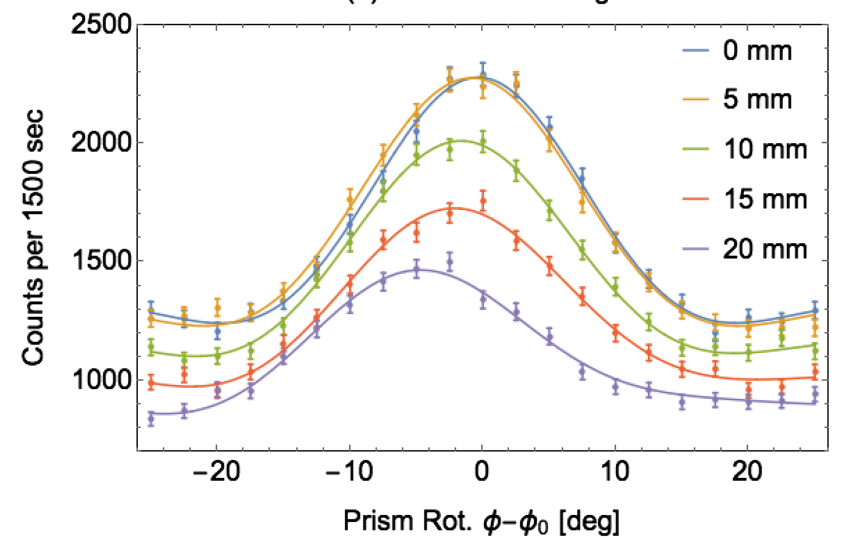

(b) After Annealing

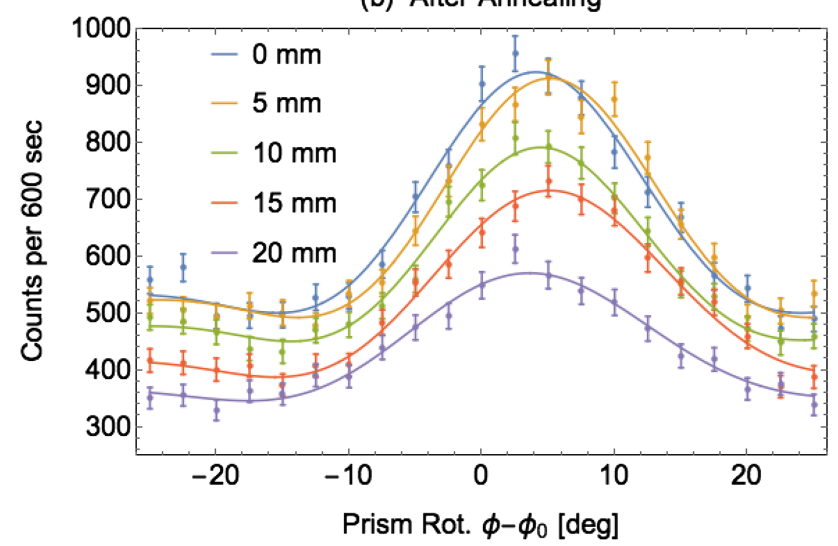

FIG. 5. The RRR peaks before (a) and after (b) annealing with best fits. Each curve is for a different translation of the interferometer relative to the incoming beam.

some portions of the interferometer. The fused silica prism was left in place while the interferometer was annealed, keeping $\delta_{p}$ constant before and after annealing. This allowed us to measure an absolute shift in the Bragg plane alignment of the splitter blade and the mirror and analyzer blades of about $100 \mathrm{nrad}$ from annealing, because $\delta_{p}$ drops out when taking the difference between $\delta_{i, j}^{\text {rel }}$ before and after annealing.

The fitted $\Delta_{i, j}$ parameters can be seen as the worsening visible distortion in the RR peak, and less so for the RRR peak, as the interferometer is translated (Figs. 4 and 5). This indicates that the thickness difference between the mirror and splitter blades changes by about $20 \%$ of the pendellösung length over $20 \mathrm{~mm}$, which corresponds to a thickness difference of about $7 \mu \mathrm{m}$, given the $34 \mu \mathrm{m}$ pendellösung length for the (111) reflection at $4.4 \AA$. The same is not true of the RRR peak, indicating that the mirror blade likely has a varying thickness. Alternatively, the mirror blade could be flat, with the splitter and analyzer blades having a similar distorted shape.

The technique of using the deflecting prism to measure Bragg plane misalignments only applies to one of the mirror blades, leaving the other unmeasured. We therefore cannot predict the contrast solely from the fits of the RR and RRR peak positions and structure. The interferometer contrast is a function of the difference in thickness between the splitter and analyzer blades and the two mirror blades separately. However, (a) Relative Misalignments

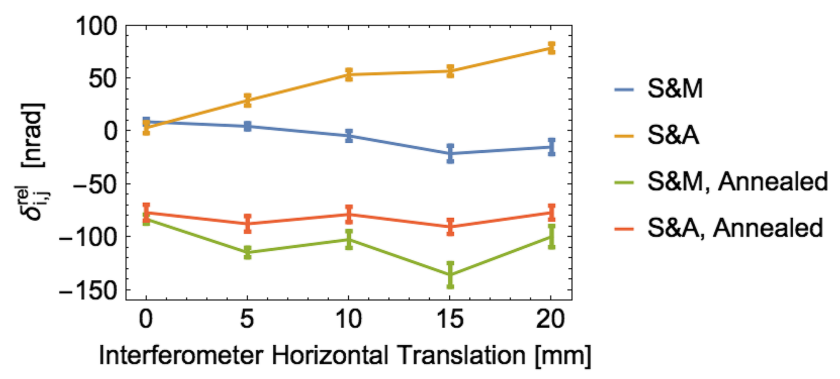

(b) Absolute Misalignments

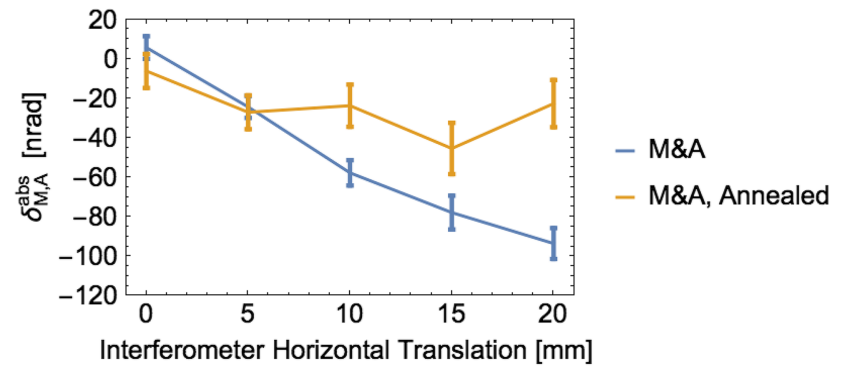

(c) Thickness Differences

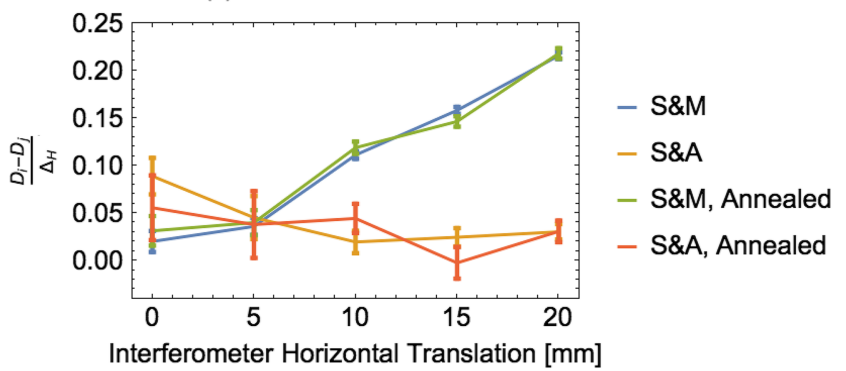

FIG. 6. Bragg plane misalignments and thickness differences before and after annealing. Shown are (a) the relative Bragg plane misalignment between the splitter and the mirror and analyzer blades, (b) the absolute misalignment between the mirror and the analyzer blades, and (c) the thickness differences as a function of interferometer translation. $S, M$, and $A$ refer to the splitter, mirror, and analyzer blades, respectively.

the mean count rate of an interferogram is at its highest when all four diffracting crystals have the same thickness.

Before annealing, there was no contrast visible at $2.2 \AA$ or $4.4 \AA$. After annealing, the interferometer was tested at $4.4 \AA$ and $2.7 \AA$. There was up to $20 \%$ contrast observed at $4.4 \AA$ after annealing. The contrast at $2.7 \AA$ was excellent, improving from $23 \% 29$ to $90 \%$. A contrast map is shown in Fig. 7. Also shown in Fig. 7 is a contrast map of the previously highestcontrast interferometer at NIST. While the peak contrasts are similar, the range over which the contrast is high is larger for the annealed interferometer. This implies that the annealed interferometer may be especially useful for phase imaging, where the incoming beam is much larger (typically $\sim 1 \mathrm{~cm}$ in diameter).

The better contrast at $2.7 \AA$, compared to that at $4.4 \AA$, for the annealed interferometer is likely due in part to the better vibrational and environmental isolation provided by the different facility. The more severe Bragg angle at $4.4 \AA$ ( $44.5^{\circ}$ versus $25.6^{\circ}$ ) also creates a larger path separation, rendering the interferometer more sensitive to vibrations. However, the dependence of contrast on wavelength may also be due in part 

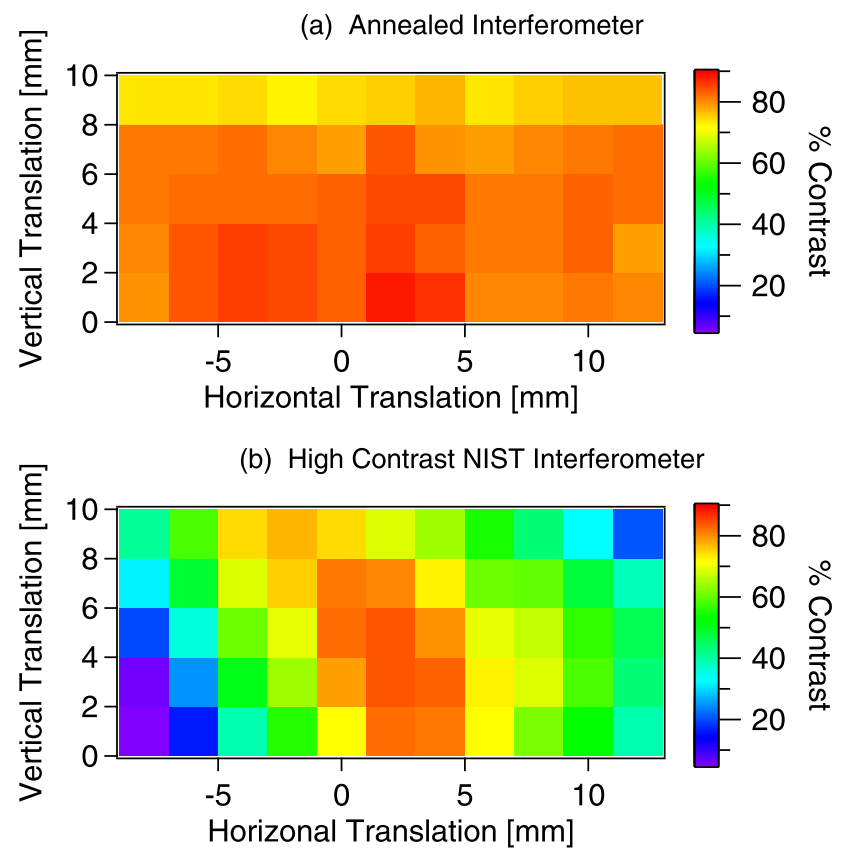

FIG. 7. Contrast Maps at $2.7 \AA$ for (a) the annealed interferometer and for (b) the previously highest-contrast NIST interferometer. The incoming beam passes through a $2 \mathrm{~mm} \times 8 \mathrm{~mm}$ slit in both cases. The peak contrast of the annealed interferometer is only slightly higher, but it shows high contrast over a wider spatial range.

to the thickness variations across the interferometer blades. It is conceivable that the demonstrated thickness variation in the mirror blade that we measured at $4.4 \AA$ [Fig. 6(c)] is less pronounced for the $2.7 \AA$ beam geometry.

\section{CONCLUSIONS}

We have shown that annealing a neutron interferometer can refine Bragg plane misalignments enough to drastically improve the contrast. While the Bragg plane alignment can be improved by annealing, the only way to fix the thickness variations in the interferometer blades would be to remachine and etch the interferometer again. The variation in the blade thickness is believed to be a principle cause of the lower contrast at $4.4 \AA$, when compared to that at $2.7 \AA$ for the interferometer annealed in this work. It is possible that with the addition of annealing treatments to the neutron interferometer postmachining fabrication process, less etching will be required. If this is the case, then the annealing step may also prevent the thickness variation in the crystal blades caused by deep etching depths, resulting in higher quality interferometers with blades that are more uniform and parallel and that have better Bragg plane alignment.

\section{ACKNOWLEDGMENTS}

The authors would like to thank Juscelino Leao for helping them with the annealing furnace and John Barker for discussing the annealing of silicon and crystal quality with them. This work was supported in part by the US Department of Energy under Grant No. DE-FG02-97ER41042, National Science Foundation Grant Nos. PHY-1307426 and PHY-1205342, Canadian Excellence Research Chairs (CERC) (No. 215284), the Canada First Research Excellence Fund (CFREF), Natural Sciences and Engineering Research Council of Canada (NSERC) Discovery (No. RGPIN-418579), Collaborative Research and Training Experience (CREATE) (No. 414061), and the NIST Quantum Information Program.

${ }^{1}$ H. Rauch, W. Treimer, and U. Bonse, Phys. Lett. A 47, 369 (1974).

${ }^{2}$ R. Colella, A. W. Overhauser, and S. A. Werner, Phys. Rev. Lett. 34, 1472 (1975).

${ }^{3}$ H. Rauch, A. Zeilinger, G. Badurek, A. Wilfing, W. Bauspiess, and U. Bonse, Phys. Lett. A 54, 425 (1975).

${ }^{4}$ S. A. Werner, R. Colella, A. W. Overhauser, and C. Eagen, Phys. Rev. Lett. 35, 1053 (1975)

${ }^{5}$ Y. Hasegawa, R. Loidl, G. Badurek, M. Baron, and H. Rauch, Nature 425, 45 (2003).

${ }^{6}$ F. Pfeiffer, C. Grünzweig, O. Bunk, G. Frei, E. Lehmann, and C. David, Phys. Rev. Lett. 96, 215505 (2006).

${ }^{7}$ C. W. Clark, R. Barankov, M. G. Huber, M. Arif, D. G. Cory, and D. A. Pushin, Nature 525, 504 (2015).

${ }^{8}$ D. Sarenac, M. G. Huber, B. Heacock, M. Arif, C. W. Clark, D. G. Cory, C. B. Shahi, and D. A. Pushin, Opt. Express 24, 22528 (2016).

${ }^{9}$ H. Lemmel, P. Brax, A. Ivanov, T. Jenke, G. Pignol, M. Pitschmann, T. Potocar, M. Wellenzohn, M. Zawisky, and H. Abele, Phys. Lett. B 743, 310 (2015).

${ }^{10}$ K. Li, M. Arif, D. Cory, R. Haun, B. Heacock, M. Huber, J. Nsofini, D. Pushin, P. Saggu, D. Sarenac et al., Phys. Rev. D 93, 062001 (2016).

${ }^{11} \mathrm{H}$. Rauch and S. A. Werner, Neutron Interferometry: Lessons in Experimental Quantum Mechanics, Wave-Particle Duality, and Entanglement (Oxford University Press, 2015), Vol. 12.

${ }^{12}$ M. Zawisky, J. Springer, R. Farthofer, and U. Kuetgens, Nucl. Instrum. Methods Phys. Res., Sect. A 612, 338 (2010).

${ }^{13}$ B. Heacock, M. Arif, R. Haun, M. G. Huber, D. A. Pushin, and A. R. Young, Phys. Rev. A 95, 013840 (2017).

${ }^{14}$ J. Arthur, C. Shull, and A. Zeilinger, Phys. Rev. B 32, 5753 (1985).

${ }^{15}$ H. Rauch, E. Seidl, D. Tuppinger, D. Petrascheck, and R. Scherm, Z. Phys. B Condens. Matter 69, 313 (1987).

${ }^{16}$ V. F. Sears, Neutron Optics: An Introduction to the Theory of Neutron Optical Phenomena and Their Applications (Oxford University Press, USA, 1989), Vol. 3.

${ }^{17}$ H. Lemmel, Acta Crystallogr., Sect. A: Found. Crystallogr. 69, 459 (2013).

${ }^{18}$ J. Nsofini, K. Ghofrani, D. Sarenac, D. Cory, and D. Pushin, Phys. Rev. A 94, 062311 (2016).

${ }^{19}$ J. Nsofini, D. Sarenac, K. Ghofrani, M. G. Huber, M. Arif, D. G. Cory, and D. A. Pushin, J. Appl Phys. 122, 054501 (2017).

${ }^{20}$ U. Bonse, W. Graeff, R. Teworte, and H. Rauch, Phys. Status Solidi A 43, 487 (1977).

${ }^{21}$ D. Petrascheck and H. Rauch, Acta Crystallogr., Sect. A: Found. Crystallogr. 40, 445 (1984).

${ }^{22}$ H. Lemmel, Phys. Rev. B 76, 144305 (2007).

${ }^{23}$ T. Potocar, M. Zawisky, H. Lemmel, J. Springer, and M. Suda, Acta Crystallogr., Sect. A: Found. Adv. 71, 534 (2015).

${ }^{24}$ J. Nico, M. Arif, M. Dewey, T. Gentile, D. Gilliam, P. Huffman, D. Jacobson, and A. Thompson, J. Res. Natl. Inst. Stand. Technol. 110, 137 (2005).

${ }^{25}$ C. Shahi, M. Arif, D. Cory, T. Mineeva, J. Nsofini, D. Sarenac, C. Williams, M. Huber, and D. Pushin, Nucl. Instrum. Methods Phys. Res., Sect. A 813, 111 (2016).

${ }^{26}$ D. Pushin, M. Huber, M. Arif, C. Shahi, J. Nsofini, C. Wood, D. Sarenac, and D. Cory, Adv. High Energy Phys. 2015, 1-7.

${ }^{27}$ R. Newman, Rep. Prog. Phys. 45, 1163 (1982).

${ }^{28}$ W. Reimers, H.-A. Crostack, M. Wrobel, and G. Eckold, Measurement of Residual and Applied Stress Using Neutron Diffraction (Springer, 1992), pp. 263-276.

${ }^{29}$ C. J. Wood, M. O. Abutaleb, M. G. Huber, M. Arif, D. G. Cory, and D. A. Pushin, Phys. Rev. A 90, 032315 (2014). 